Draft Version September 9, 2018

Preprint typeset using LATEX style emulateapj v. 12/01/06

\title{
A CORE-COLLAPSE SUPERNOVA MODEL FOR THE EXTREMELY LUMINOUS TYPE IC SUPERNOVA 2007BI: AN ALTERNATIVE TO THE PAIR-INSTABILITY SUPERNOVA MODEL
}

\author{
Takashi Moriya $^{1,2,3}$, Nozomu Tominaga ${ }^{4,1}$, Masaomi Tanaka ${ }^{1}$, Keilchi Maeda ${ }^{1}$, and Ken'ichi Nomoto ${ }^{1,2}$ \\ Draft version September 9, 2018
}

\begin{abstract}
We present a core-collapse supernova model for the extremely luminous Type Ic supernova $2007 \mathrm{bi}$. By performing numerical calculations of hydrodynamics, nucleosynthesis, and radiation transport, we find that SN 2007bi is consistent with the core-collapse supernova explosion of a $43 M_{\odot}$ carbon and oxygen core obtained from the evolution of a progenitor star with a main sequence mass of $100 M_{\odot}$ and metallicity of $Z=Z_{\odot} / 200$, from which its hydrogen and helium envelopes are artificially stripped. The ejecta mass and the ejecta kinetic energy of the models are $40 M_{\odot}$ and $3.6 \times 10^{52}$ erg. The ejected ${ }^{56} \mathrm{Ni}$ mass is as large as $6.1 M_{\odot}$, which results from the explosive nucleosynthesis with large explosion energy. We also confirm that SN 2007bi is consistent with a pair-instability supernova model as has recently been claimed. We show that the earlier light curve data can discriminate between the models for such luminous supernovae.

Subject headings: supernovae: general, supernovae: individual (SN 2007bi, SN 2006gy), gamma-ray burst: general
\end{abstract}

\section{INTRODUCTION}

A massive star with the main sequence mass $\left(M_{\mathrm{MS}}\right)$ in the range of $10-140 M_{\odot}$ forms an Fe core in its center and eventually collapses. This collapse is thought to end up with the core-collapse supernova (SN) of Type II, Ib, or Ic (Filippenko 1997 for a review). If a star is as massive as $M_{\mathrm{MS}}=140-300 M_{\odot}$, the oxygen-rich core becomes dynamically unstable owing to the electronpositron pair creations (Rakavy \& Shaviv 1967; Barkat, Rakavy, \& Sack 1967). As the internal energy is spent by the pair creations, the core loses the stability and starts to collapse. When the central temperature exceeds $\sim 5 \times 10^{9} \mathrm{~K}$, the core becomes stable but the temperature is so high that oxygen burning becomes explosive to produce enough energy to unbind the star. A large amount of ${ }^{56} \mathrm{Ni}$ is synthesized by the explosive burning (e.g., Umeda \& Nomoto 2002, hereafter UN02; Heger \& Woosley 2002) and the subsequent radioactive decays power the light curve. Thus this event is theoretically predicted to be observed as a pair-instability supernova (PISN). Some luminous SNe like SN 2006gy (Ofek et al. 2007; Smith et al. 2007) have been suggested to be PISNe (see Section 4.2), but no clear consensus has been reached (e.g., Kawabata et al. 2009).

Recently, Gal-Yam et al. (2009) (G09 hereafter) suggested that the extremely luminous Type Ic SN 2007bi is the first observed example of the PISN. They showed that the PISN model is consistent with the observed light curve (LC) and the nebular spectra of SN 2007bi. They estimated the masses of $\mathrm{C}, \mathrm{O}, \mathrm{Na}, \mathrm{Mg}, \mathrm{Ca}$, and ${ }^{56} \mathrm{Ni}$ from the observed optical spectra. Other elements

\footnotetext{
1 Institute for the Physics and Mathematics of the Universe, University of Tokyo, Kashiwanoha 5-1-5, Kashiwa, Chiba 277-8583, Japan; takashi.moriya@ipmu.jp

2 Department of Astronomy, Graduate School of Science, University of Tokyo, Bunkyo-ku, Tokyo 113-0033, Japan

3 Recearch Center for the Early Universe, Graduate School of Science, University of Tokyo, Bunkyo-ku, Tokyo 113-0033, Japan

4 Department of Physics, Faculty of Science and Engineering Konan University, 8-9-1 Okamoto, Kobe, Hyogo 658-8501, Japan
}

with no strong emission lines in the optical range, $\mathrm{Si}$ and $\mathrm{S}$, are assumed to be the same as the PISN model of Heger \& Woosley (2002). Young et al. (2010) (Y10 hereafter) showed multi-color observations of SN 2007bi and the metallicity of the host galaxy. However, the above observations of SN 2007bi have not quantitatively been compared with the core-collapse SN models. In view of the importance of clarifying the final fates of very massive stars, we examine how strong the observational constraint on the theoretical models are.

The aim of this Letter is to show that a core-collapse SN model is indeed consistent with the observed properties of SN 2007bi, if the progenitor is as massive as $M_{\mathrm{MS}} \sim 100 M_{\odot}$ and the explosion energy is large. This would imply that SN 2007bi might not necessarily be a PISN. In Section 2, we summarize the progenitor models for SN 2007bi and numerical methods used for our calculations of hydrodynamics, nucleosynthesis, and the LC. The core-collapse SN models of SN 2007bi are presented in Section 3 and the results are discussed in Section 4.

\section{PROGENITOR AND EXPLOSION MODELING}

\subsection{Progenitor}

The high peak luminosity and the long rise time of the LC of SN 2007bi (G09, Y10) require a large amount of ${ }^{56} \mathrm{Ni}\left(>3 M_{\odot}, \mathrm{G} 09\right)$ and a large ejecta mass. These observations imply that the progenitor of SN 2007bi is massive. We apply a pre-SN model with $M_{\mathrm{MS}}=100 M_{\odot}$ calculated by Umeda \& Nomoto (2008, UN08 hereafter). UN08 assumed the metallicity of the progenitor models to be $Z=Z_{\odot} / 200$, which is small enough to avoid a large amount of wind mass loss. Then the pre-SN model remains as massive as $M=83 M_{\odot}$, whose carbon + oxygen $(\mathrm{C}+\mathrm{O})$ core is massive enough $\left(43 M_{\odot}\right)$ to produce a large amount of ${ }^{56} \mathrm{Ni}$.

However, the pre-SN model has a massive H-rich envelope, while SN 2007bi does not show the lines of either $\mathrm{H}$ or He. Therefore the progenitor must have lost its $\mathrm{H}$ rich envelope $\left(36 M_{\odot}\right)$ and He layer $\left(4 M_{\odot}\right)$ during the pre-SN evolution, thus having only the bare $\mathrm{C}+\mathrm{O}$ core at 


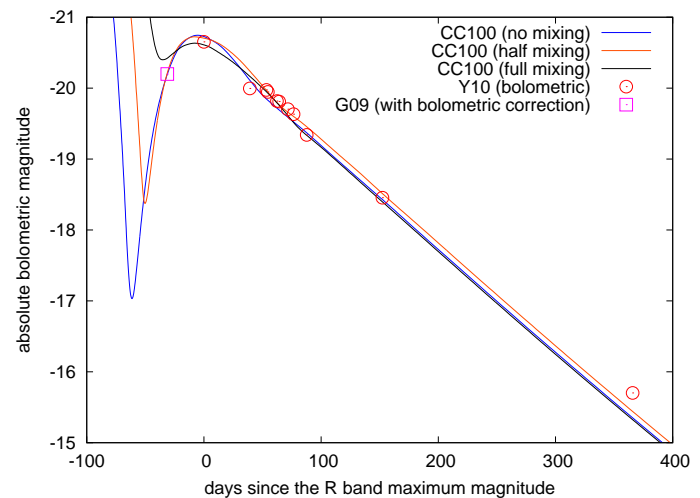

FIG. 1. - Bolometric LCs of the $\mathrm{C}+\mathrm{O}$ star SN models CC100 $\left(M_{\mathrm{ej}}=40 M_{\odot}, E_{\mathrm{kin}}=3.6 \times 10^{52} \mathrm{erg}\right.$, and $\left.M_{56 \mathrm{Ni}}=6.1 M_{\odot}\right)$. The observed bolometric LC (open circles) is taken from Y10. The bolometric magnitude of the rise part of SN 2007bi (open square) is estimated from the $R$ band magnitude. All the calculated LC has the same physical structure but the degrees of mixing are different. The horizontal axis shows the days in the rest frame.

the explosion. We construct the pre-SN $\mathrm{C}+\mathrm{O}$ star model of $43 M_{\odot}$, by removing the H-rich envelope and He layer from the $83 M_{\odot}$ star. Note that the metallicity of the host galaxy of SN 2007bi $\left(Z \sim Z_{\odot} / 3\right.$, Y10) is higher than that of our adopted progenitor $\left(Z=Z_{\odot} / 200\right)$. The wind mass loss is expected to work more efficiently and the main sequence mass of the progenitor which has the $\mathrm{C}+\mathrm{O}$ core mass of $43 M_{\odot}$ might be more massive. The rotation of stars can also play a role in the mass loss (e.g., Meynet et al. 2003; Hirschi et al. 2004; Maeder et al. 2005; Georgy et al. 2009). Another possible cause of such envelope stripping is the formation of a common envelope during a close binary system, where the smaller mass companion star spirals into the envelope of the more massive star. The outcome depends on whether the energy available from the spiral-in exceeds the binding energy of the common envelope, thus being either a merging of the two stars or the formation of two compact stars, e.g., a $\mathrm{C}+\mathrm{O}$ star and a $\mathrm{He}$ star.

\subsection{Explosion Modeling}

We calculate the explosion of the pre-SN $\mathrm{C}+\mathrm{O}$ star $\left(43 M_{\odot}\right)$ as described above. Explosions are induced by a thermal bomb and followed by a one-dimensional Lagrangian code with the piecewise parabolic method (Colella \& Woodward 1984). Note that the explosion energy is a free parameter in core-collapse SN explosion models while it is not in PISN explosion models. Explosive nucleosynthesis is calculated as post-processing for the thermodynamical history obtained by the hydrodynamical calculations. The resultant abundance distribution is basically very similar to those calculated by UN08 (see Figures 5 and 6 in UN08).

The dynamics of the ejecta is followed until 1 day after the explosion, when the expansion already becomes homologous $(r \propto v)$. The bolometric LCs are calculated for the homologous ejecta by using the LTE radiation transfer code (Iwamato et al. 2000) that includes the radioactive decays of ${ }^{56} \mathrm{Ni}$ and ${ }^{56} \mathrm{Co}$ as energy sources. This code calculates the $\gamma$-ray transport for a constant $\gamma$-ray opacity $\left(0.027 \mathrm{~cm}^{2} \mathrm{~g}^{-1}\right.$, Axelrod 1980) and assumes all

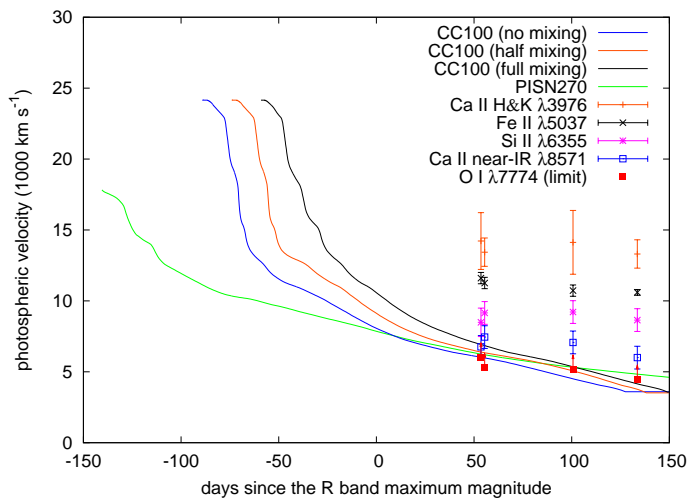

FIG. 2.- Photospheric velocities of the core-collapse SN and PISN models. The horizontal axis shows the days in the rest frame. The line velocities of SN 2007bi observed by Y10 are also shown for comparison. The line velocities of O I $\lambda 7774$ shows the lower limit. All the photospheric velocities are low enough to be consistent with the observed line velocities.

the emitted positrons are absorbed in situ ${ }^{5}$. For the optical radiation transport, the Thomson scattering opacity is obtained by calculating the electron density from the Saha equation, and the Rosseland mean opacity is estimated from the empirical relation to the Thomson scattering opacity (Deng et al. 2005).

\section{CORE-COLLAPSE SUPERNOVA MODELS FOR SN 2007BI}

We construct several core-collapse SN models and compare with the observations of the bolometric LC and the line velocities of SN 2007bi shown in Y10. Since the LC of Y10 does not cover the rising part of the LC, we estimate the bolometric magnitude of the rising part from the $R$ band observations (G09) assuming the same bolometric correction (0.45 mag) as in the $R$ band maximum. We also take into account mixing since it is possible that a jet emerges from the central remnant and causes the mixing of the ejecta (e.g., Maeda \& Nomoto 2003, Tominaga 2009).

The LCs of successful models (CC100) are shown in Figure 1. The kinetic energy $\left(E_{\text {kin }}\right)$, ejecta mass $\left(M_{\mathrm{ej}}\right)$, and ${ }^{56} \mathrm{Ni}$ mass $\left(M_{56 \mathrm{Ni}}\right)$ in the ejecta are $E_{\text {kin }}=3.6 \times 10^{52}$ erg, $M_{\mathrm{ej}}=40 M_{\odot}$, and $M_{56 \mathrm{Ni}}=6.1 M_{\odot}$, which are the same in all the models. The mass cut between the ejecta and the compact remnant is set at $M_{r}=3 M_{\odot}$, where $M_{r}$ is the mass coordinate, so that the ejecta contains $6.1 M_{\odot}$ of ${ }^{56} \mathrm{Ni}$, which turns out to be consistent with the bolometric LC of SN 2007bi. The kinetic energy $E_{\text {kin }}$ needs to be large to produce $M_{56 \mathrm{Ni}}=6.1 M_{\odot}$. The mass of some elements in the ejecta are summarized in Table 1. The kinetic energy is as large as those of previously observed SNe which were associated with a gammaray burst (SNe 1998bw, 2003dh, 2003lw; e.g., Nomoto et al. 2006).

We adopt two different degrees of mixing to see its effects on the LC. The full-mixing model assumes that the whole ejecta are uniformly mixed. The half-mixing model assumes that the inner half of the ejecta (in the mass coordinate) is uniformly mixed. One of the effects of the mixing is seen in the rise time of the LC. With

\footnotetext{
${ }^{5}$ This assumption of the positron absorption does not have much effect on the LCs we show in this paper, because the contribution from the gamma-rays is still a dominant energy source of them.
} 
TABLE 1

The Amount of Elements Contained in the Ejecta

\begin{tabular}{lcccccccc}
\hline \hline${ }^{12} \mathrm{C}$ & ${ }^{16} \mathrm{O}$ & ${ }^{20} \mathrm{Ne}$ & ${ }^{24} \mathrm{Mg}$ & ${ }^{28} \mathrm{Si}$ & ${ }^{32} \mathrm{~S}$ & ${ }^{36} \mathrm{Ar}$ & ${ }^{40} \mathrm{Ca}$ & ${ }^{56} \mathrm{Ni}$ \\
\hline 1.4 & 18.7 & 1.4 & 1.5 & 5.1 & 2.7 & 0.5 & 0.4 & 6.1 \\
\hline \multicolumn{7}{l}{ Note. - Units: $M_{\odot}$}
\end{tabular}

mixing, ${ }^{56} \mathrm{Ni}$ is distributed closer to the surface of the ejecta, so that the diffusion time is shorter and the rise time becomes shorter. The rise time of the model without mixing is 85 days while the rise times of the halfmixing model and the full-mixing model are 67 days and 52 days, respectively. As the rise time of SN 2007bi is not observationally well-determined, all the models are consistent with the bolometric LC of SN 2007bi. The initial decline part of the calculated LCs before maximum is formed by the shock heating of the envelope and its subsequent cooling due to rapid expansion. Radiation hydrodynamical calculations are required to obtain the realistic LC at this epoch.

In Figure 2, we show the photospheric velocities obtained by the LC calculations. With the photospheric velocities, we also show the observed line velocities of SN 2007bi taken from Figure 17 of Y10. The photospheric velocities of all the models are consistent with the observed lowest line velocities, which are thought to trace the photospheric velocity.

One of the big difference between the core-collapse SN models and the PISN models is the abundance of the elements like Si and S. The abundance of our core-collapse SN model is consistent with the directly estimated abundances from the one-zone model of G09 adopted to the observed emission lines, i.e., $\mathrm{C}, \mathrm{O}, \mathrm{Na}, \mathrm{Mg}, \mathrm{Ca}$, and ${ }^{56} \mathrm{Ni}$. However, it differs significantly in elements whose abundances are only indirectly constrained by the spectra (such as Si and S) as these may have played a role in line cooling processes. In order to confirm that the abundance of the core-collapse SN model are consistent with the nebular spectra, we have to perform spectral synthesis calculations for the realistic hydrodynamical model of ejecta rather than the single-zone adopted by G09. As Si and S have many emission lines in the infrared range, infrared spectra are also helpful to distinguish PISNe from core-collapse SNe. We also point out that, if SN 2007bi is confirmed to be a PISN, we could expect that PISNe played a role in the chemical enrichment in the early Universe and there should be some old stars with chemical compositions expected from PISNe, although they are still not discovered (e.g., Cayrel et al. 2004).

\section{CONCLUSIONS AND DISCUSSION}

In this Letter, we have shown that the LC and photospheric velocity of SN 2007bi are well-reproduced by the core-collapse SN model CC100. As some gamma-ray bursts are connected to such high energy Type Ic SNe, the extremely luminous SNe like SN 2007bi could also be connected to gamma-ray bursts which result from very massive stars. If this is the case, extremely luminous $\mathrm{SNe}$ like SN 2007bi could be connected to gamma-ray bursts of much more massive star origin than known SNe associated with a gamma-ray burst. Even stars more massive than $300 M_{\odot}$ could be the origin of luminous $\mathrm{SNe}($ e.g., Ohkubo et al. 2006, 2009).

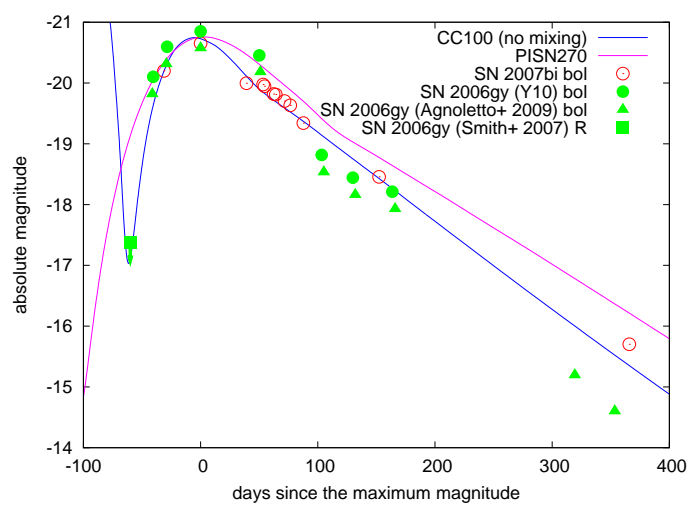

FIG. 3.- A PISN model for SN 2006gy (PISN270). The bolometric LC of SN 2006gy is taken from Y10 and Agnoletto et al. (2009). The first point of SN 2006gy is the $R$ band upper limit from Smith et al. (2007). The CC100 no-mixing model and observed bolometric LC of SN 2007bi in Figure 1 are also shown. The horizontal axis shows the days in the rest frame.

We note, however, that, although SN 2007bi may not necessarily be a PISN, the observational data available for SN 2007bi is not sufficient to single out the explosion mechanism. In fact, Kasen \& Bildsten (2009) suggested that the magnetar-powered light curve model (also, Maeda et al. 2007; Woosley 2009) might explain the LC of SN 2007bi.

Here we show the comparison between our PISN model and SN 2007bi, and discuss how to distinguish the models for luminous SNe. We also apply such LC comparison to SN 2006gy.

\subsection{Pair-Instability Supernova Models for SN $2007 b i$}

In Section 3 (Figure 1), we have shown that observations of SN 2007bi are well reproduced by the corecollapse SN model (CC100). Here we confirm the claim made by G09 that a PISN model can also be consistent with the bolometric LC of SN 2007bi by using the approximate PISN model PISN270.

The PISN270 model is constructed by scaling the physical structure of the homologously expanding model CC100 to the ejecta model with $M_{\mathrm{ej}}=121 M_{\odot}$ and $E_{\text {kin }}=7 \times 10^{52} \mathrm{erg}$. The ejecta mass $M_{\mathrm{ej}}$ is the same as the $\mathrm{C}+\mathrm{O}$ core mass of the PISN model with $M_{\mathrm{MS}}=270 M_{\odot}(\mathrm{UN} 02)$, and $E_{\mathrm{kin}}$ is obtained from the nuclear energy released by explosive nuclear burning of the $\mathrm{C}+\mathrm{O}$ core (UN02). Here the same amount of ${ }^{56} \mathrm{Ni}\left(M_{56 \mathrm{Ni}}=9.8 M_{\odot}\right)$ as in the $270 M_{\odot}$ model (UN02) is assumed to be synthesized in the inner layers. Note that the $270 M_{\odot}$ model of UN02 still has the Hrich and He envelopes at the time of explosion and, here, we assume that the envelopes were stripped off by some mechanism.

Figure 3 shows that the bolometric LC of PISN270 (the red line) is consistent with the bolometric LC of SN 2007bi (red open circles). The rise time to the LC peak for PISN270 is $\sim 150$ days, being consistent with the PISN model in G09. This rise time is longer than the core-collapse SN model CC100 (Figure 3), because the photon diffusion takes more time in more massive PISN270. Although $M_{56 \mathrm{Ni}}$ of PISN270 is $\sim 1.6$ times larger than that of the core-collapse SN CC100 model, the longer rise time lowers the peak brightness powered by the radioactive decay. These two effects make the 
peak magnitude of PISN270 similar to CC100.

This difference in the rising part of the LC is important to discriminate between the core-collapse SN and the PISN models. Although SN 2007bi was not observed early enough, much earlier observations before the peak could constrain the SN type from the LC. In addition, as already mentioned in Section 3, the abundance of $\mathrm{Si}$ and $\mathrm{S}$ would also be a key to distinguish between the two models.

\subsection{Models for SN 2006gy}

As mentioned in Section 1, there has been some suggestions that the luminous Type IIn SN 2006gy is a PISN (e.g., Smith et al. 2007). We thus apply our LC models for comparison with SN 2006gy. Figure 3 shows the bolometric LC of SN 2006gy (filled circles and triangles). As only the $R$ band magnitude was observed in the early epochs of SN 2006gy (the filled square), we cannot construct the bolometric LC at the early epochs but we can constrain the rise time of the LC. Our calculations show that the rise time of the PISN model is too slow to be consistent with SN 2006gy. Although our PISN model does not have a H-rich envelope, the presence of the $\mathrm{H}$ rich envelope could even slow down the brightening (e.g., Kawabata et al. 2009). Woosley et al. (2008) showed that the interaction between the pulsating core and the envelope can power the LC of SN 2006gy. As a similar mechanism, the interaction of a SN ejecta with its very dense circumstellar matter could convert the kinetic energy of ejecta directly to radiation energy and could also be the origin a luminous SN like SN 2006gy.

We thank the anonimous referees for their advice which improved the text. Numerical calculations were carried out on the general-purpose PC farm at Center for Computational Astrophysics, CfCA, of National Astronomical Observatory of Japan. This research has been supported in part by World Premier International Research Center Initiative, MEXT, and by the Grant-in-Aid for Scientific Research of the JSPS (18104003, 20540226, 20840007) and MEXT (19047004, 22012003), Japan.

\section{REFERENCES}

Agnoletto, I., et al. 2009, ApJ, 691, 1348

Axelrod, T. S. 1980, Ph.D. thesis, Univ. California, Santa Cruz

Barkat, Z., Rakavy, G., \& Sack, N. 1967, Physical Review Letters, 18,379

Cayrel, R., et al. 2004, A\&A, 416, 1117

Colella, P., \& Woodward, P. R. 1984, J. Comput. Phy., 54, 174

Deng, J., Tominaga, N., Mazzali, P. A., Maeda, K., \& Nomoto, K. 2005, ApJ, 624, 898

Filippenko, A. V. 1997, ARA\&A, 35, 309

Gal-Yam, A., et al. 2009, Nature, 462, 624 (G09)

Georgy, C., Meynet, G., Walder, R., Folini, D., \& Maeder, A. 2009, 502,611

Heger, A., \& Woosley, S. E. 2002, ApJ, 567, 532

Hirschi, R., Meynet, G., \& Maeder, A. 2004, A\&A, 425, 649

Iwamoto, K., et al. 2000, ApJ, 534, 660

Kasen, D., \& Bildsten, L. 2009, arXiv:0911.0680

Kawabata, K. S., Tanaka, M., Maeda, K., Hattori, T., Nomoto, K., Tominaga, N., \& Yamanaka, M. 2009, ApJ, 697, 747

Maeda, K., \& Nomoto, K. 2003, ApJ, 598, 1163

Maeda, K., et al. 2007, ApJ, 666, 1069
Maeder, A., \& Meynet, G. 2005, A\&A, 429, 581

Meynet, G., \& Maeder, A. 2003, A\&A, 404, 975

Nomoto, K., Tominaga, N., Tanaka, M., Maeda, K., Suzuki, T., Deng, J. S., \& Mazzali, P. A. 2006, Nuovo Cimento B Serie, 121, 1207

Ofek, E. O., et al. 2007, ApJ, 659, L13

Ohkubo, T., Umeda, H., Maeda, K., Nomoto, K., Suzuki, T., Tsuruta, S., \& Rees, M. J. 2006, ApJ, 645, 1352

Ohkubo, T., Nomoto, K., Umeda, H., Yoshida, N., \& Tsuruta, S. 2009, ApJ, 706, 1184

Rakavy, G., \& Shaviv, G. 1967, ApJ, 148, 803

Smith, N., et al. 2007, ApJ, 666, 1116

Tominaga, N. 2009, ApJ, 690, 526

Umeda, H., \& Nomoto, K. 2002, ApJ, 565, 385 (UN02)

Umeda, H., \& Nomoto, K. 2008, ApJ, 673, 1014 (UN08)

Woosley, S. E., Blinnikov, S., \& Heger, A. 2007, Nature, 450, 390

Woosley, S. E. 2009, arXiv:0911.0698

Young, D. R., et al. 2010, A\&A, 512, A70 (Y10) 